\title{
Any Sense in Classroom Scents? Aroma of Rosemary Essential Oil Significantly Improves Cognition in Young School Children
}

\author{
Mark Moss*, Victoria Earl, Lucy Moss, Tom Heffernan \\ Department of Psychology, Faculty of Health and Life Sciences, Northumbria University, Newcastle upon Tyne, United Kingdom \\ Email: ${ }^{\star}$ mark.moss@unn.ac.uk
}

How to cite this paper: Moss, M., Earl, V., Moss, L. and Heffernan, T. (2017) Any Sense in Classroom Scents? Aroma of Rosemary Essential Oil Significantly Improves Cognition in Young School Children. Advances in Chemical Engineering and Science, 7, 450463.

https://doi.org/10.4236/aces.2017.74032

Received: September 1, 2017

Accepted: October 27, 2017

Published: October 30, 2017

Copyright $\odot 2017$ by authors and Scientific Research Publishing Inc. This work is licensed under the Creative Commons Attribution International License (CC BY 4.0).

http://creativecommons.org/licenses/by/4.0/

\begin{abstract}
The inhalation of the aroma of Rosemary essential oil has been shown to enhance cognition in healthy adults. In this independent groups design study we exposed forty schoolchildren aged nine to eleven years to either Rosemary aroma or no aroma in a classroom setting where they completed standardised tests of working memory in fifteen minute procedure. Analysis of the data revealed that performance on the Immediate serial recall, Sentence span, and Counting span tasks were significantly better in the Rosemary aroma condition and possessed medium to large effect sizes. This is the first study to demonstrate such effects in children and suggests that the potential for enhancement is greater than in adults. The findings are discussed in terms of the potential for improving academic attainment through natural interventions and the possible mechanisms behind such effects.
\end{abstract}

\section{Keywords}

Rosemary, Aroma, Working Memory, Children

\section{Introduction}

Plant extracts and essential oils have been used in medicine, spiritual rituals, and social events and occasions since before the advent of written history [1]. The aromas of such products have an equally well documented record of application in religious, environmental and personal domains, and for a wide range of purported benefits [2]. In the contemporary domain, evidence exists for the beneficial use of aromas to change smoking behaviours [3] aid weight loss during dieting [4], and moderating blood pressure via aromatherapy massage to the feet [5]. In addition to the influence on aspects of health and wellbeing a number of 
studies have investigated possible effects on cognition. Warm and colleagues [6] demonstrated that Peppermint (Mentha piperita) aroma could enhance vigilance performance independent of subjective evaluations of stress during task performance. In contrast Peppermint aroma has been demonstrated to positively impact on both cognition and changes in pre- to post-testing mood state when compared to Ylang Ylang [7]. Clearly, the type of cognitive assessments, subjective variables and the timing of their recording play important roles in the pattern of effects observed.

One aroma that has received attention is that of Rosemary (Rosmarinus officinalis). With a reputation for its beneficial effects on memory [8] it is perhaps not surprising that interest has gathered on the potential for Rosemary aroma to enhance cognition. An early investigation reported that Rosemary not only improved cognition-as assessed through mathematical calculations-but also impacted on subjective reports of mood that were further reflected in EEG waveform measurements [9]. A subsequent computerised cognitive assessment of the effects of exposure to Rosemary aroma identified positive effects on long term memory [10], a finding later supported by investigations into prospective memory [11]. The effects reported in these studies were observed in adults aged 18 to 35 years, and an interesting question can be focussed around the potential for effects outside of this age group. A Cochrane review of aromatherapy exists for its use in dementia [12], and a small number of papers review treatment effects for non-dementia age related ailments [13] [14]. With regard to younger populations aromatherapy has been examined as a possible intervention for sleep problems in autism [15], anxiety during stem cell infusion [16] and dental treatment [17], and as a complementary treatment for chronic pain [18]. In addition, an interesting odour association effect has been demonstrated in school children [19]. Five-year-olds were given the experience of failure on a cognitive maze in a room scented with fragrance and later given another cognitively challenging test in a different room scented with either the same odour, a different odour, or no odour. Children who performed the test in the presence of the same odour as the maze task did significantly worse than subjects in any other group. This association however does not represent the impact of the odour independently and it is such effects that are of interest here. A review of the general literature concludes that the use of aromas in the classroom could enhance performance via changes in alertness [20], but does not cite any experimental demonstrations in School settings. We aim to address this in the current study.

Human memory is usually disaggregated to a series of subsystems and processes defined by long term memory and the tripartite system of working memory that can be conceptualised as consisting of a central executive and two semi-independent slave systems; the visuospatial sketch pad and phonological loop [21]. Importantly for the developing child a body of research has investigated the importance of working memory and its components in relation to scholastic attainment [22] [23]. Exploration has been made into working memory training to improve performance in children across the ability spectrum 
[24], with clear suggestions that as a predictor of subsequent achievement working memory is better than IQ [25], especially for those with learning difficulties [26] [27].

Given the importance of working memory in the educational domain it is perhaps not surprising that potential interventions to augment performance have been investigated. Physical activity that improves cardiorespiratory fitness has been shown to improve working memory performance in pre-adolescents [28]. The impact of breakfast and diet have also been the subject of attention, and although the findings are clear in respect the negative impact of poor diet [29] [30], the identification of the best breakfast to enhance performance has proven more difficult [31] [32] [33]. A recent review of micronutrient supplementation studies concluded that positive effects were only to be found for children deficient as a consequence of diet, with no overall enhancement effects observed [34].

Interestingly, there appear to be no published trials investigating the potential effects of ambiently diffused aromas of essential oils on children's working memory performance. Given that a potential for enhancement exists based on the adult aroma literature, and that any improvement could feed into later scholastic attainment this seems somewhat surprising. It is the purpose of the current study to begin to address this, with an investigation of the effect of the aroma of Rosemary essential oil on aspects of working memory in children aged nine to eleven years.

\section{Method}

\subsection{Design}

A one factor independent groups experimental design was employed. The independent variable was the presence or absence of the ambient aroma of Rosemary. The dependent variables were the working memory measures of Immediate Serial Word Recall, Digit Span, Sentence Span and Counting Span and the long term memory measure Delayed Word Recall.

\subsection{Participants}

A total of forty year six pupils attending a Primary School in the North East of England volunteered to take part in the study. Parental consent was obtained using an "opt out" methodology following written communication to all parents and guardians regarding the nature and aims of the study. None of the children were withdrawn from the study based on this process. All participants were randomly allocated to one of two conditions. The Rosemary group consisted of 11 boys and 9 girls, $\mathrm{M}$ age $=10.8 \mathrm{yrs}, \mathrm{SD}=0.7$. The no aroma control group consisted of 11 boys and 9 girls, $\mathrm{M}$ age $=10.9 \mathrm{yrs}, \mathrm{SD}=0.8$.

\subsection{Testing Room}

The room used for all testing was quiet and separate from the main classrooms. It measured $4 \mathrm{~m}$ long $\times 4 \mathrm{~m}$ wide $\times 2.4 \mathrm{~m}$ high with a window stretching along 
one wall, and contained one round table and two chairs and a filing cabinet. The walls were bare save for a clock and a blackboard.

\subsection{Materials and Tasks}

The Rosemary aroma was produced by diffusing six drops of "Tisserand" organic Rosemary essential oil (First Natural Brands Ltd., Brighton, UK) using a Tisserand Aroma Stream Difuser set to the high fan setting for a period of ten minutes prior to testing.

The Immediate Serial Recall Task consisted of three trials. Each trial consisted of the oral presentation of six high frequency single syllable words at a rate of one every two seconds. A typical word list was "mouse, girl, box, hand, moon, dog". No word appeared in more than one trial. For each trial participants are required to recall the words in the correct serial order. The number of correctly recalled words was recorded. This task is designed to provide a measure of phonological working memory [35].

The Digit Span Task comprised of sets of three successive trials. Each trial consisted of the oral presentation three numbers ranging from 1 to 16 eg 3, 12, 5 . Participants are required to recall the digits in the correct order for each trial and if successful the next set of three trials increased the numbers presented by one. When the participants failed to recall in correct serial order less than two trials in a set the testing stopped and the recorded digit span was the maximum list length at which two out of the three trials were correct. This task is considered a good measure of phonological working memory with a central executive component [36].

The Sentence Span Task was adapted from the Listening Span Task [37] using simple sentences of less than ten words, presented orally in sets of increasing size beginning with two sentences. The participant is asked to judge the veracity of each sentence in turn and then to recall in any order the last word in each sentence. Testing was stopped when the child failed to correctly recall the last word in every sentence in the set. The number of sentences per trial previous to this was scored as the child's sentence span. This task is designed to tap into phonological loop and central executive functions of working memory [38].

The Counting Span Task consisted of an A5 sized booklet containing white pages on which were printed arrays of red dots. The number of dots per page ranged from 3 to 6 and was sequenced through the booklet in a randomly determined order. Participants were required to count the dots on three successive pages and then recall the number on each in correct serial order. The task was then repeated twice and if successful the next set of trials consisted four pages of dots, and so on. Testing stopped when the child failed to recall in correct serial order the number of dots on two pages or more in two trials of any given level. The child's counting span was the maximum number of pages per trial from the level previous to this. The counting span task has been shown to be effective in tapping the central executive function of working memory [38].

The Delayed Word Recall Task required participants to think back to the first 
memory game they took part in and recall in any order any of the words from that game. Number of correctly recalled items was recorded. This task is designed to measure long term verbal recall [36].

\subsection{Procedure}

The study gained approval from the Faculty of Health and Life Sciences ethics committee at Northumbria University, Newcastle, UK. In the Rosemary aroma condition four drops of essential oil were placed on the pad of the diffuser which was then placed out of sight and switched on for five minutes prior to testing each participant. On completion of the testing day the window was opened to refresh the air for the following day. The children were tested individually, seated at the table opposite the researcher. After introducing herself to the child the researcher said "you are here to play some memory games. Please don't be nervous but try the best you can to remember what I ask you to". At this point the child was given the opportunity to withdraw from the study if they felt uncomfortable continuing. The tasks were then completed in the same order for each child; Immediate Serial Recall, Digit Span, Sentence Span, Counting Span, Delayed Word Recall. The testing procedure took approximately 15 minutes per child. When all the children had been tested the class were debriefed and any questions answered.

\section{Results}

The data consisted of five memory scores for each of the forty participants. Analysis of the data was performed in SPSS version 22 using independent samples t-tests and the calculation of Cohen's d measures of effect size for each variable.

\subsection{Immediate Serial Recall}

Analysis revealed a significant difference between groups $\mathrm{t}(38)=2.53, p<0.05$. Comparison of the means indicated that children in the Rosemary aroma condition (mean $=4.60$ words) scored significantly higher than those in the no aroma control condition (mean $=3.37$ words), Cohen's $d=1.1$ representing a large effect size (Figure 1).

\subsection{Digit Span}

Analysis revealed no significant difference between groups $\mathrm{t}(38)=1.36, p=$ 0.18 . Although not significant, comparison of the means indicated that children in the Rosemary aroma condition (mean $=5.20$ digits) recalled numerically more digits correctly than those in the no aroma control condition (mean $=4.85$ digits), with a Cohen's $\mathrm{d}=0.35$ representing a small to medium effect size (Figure 2).

\subsection{Sentence Span}

Analysis revealed a significant difference between groups $\mathrm{t}(38)=2.51, p<0.05$. 


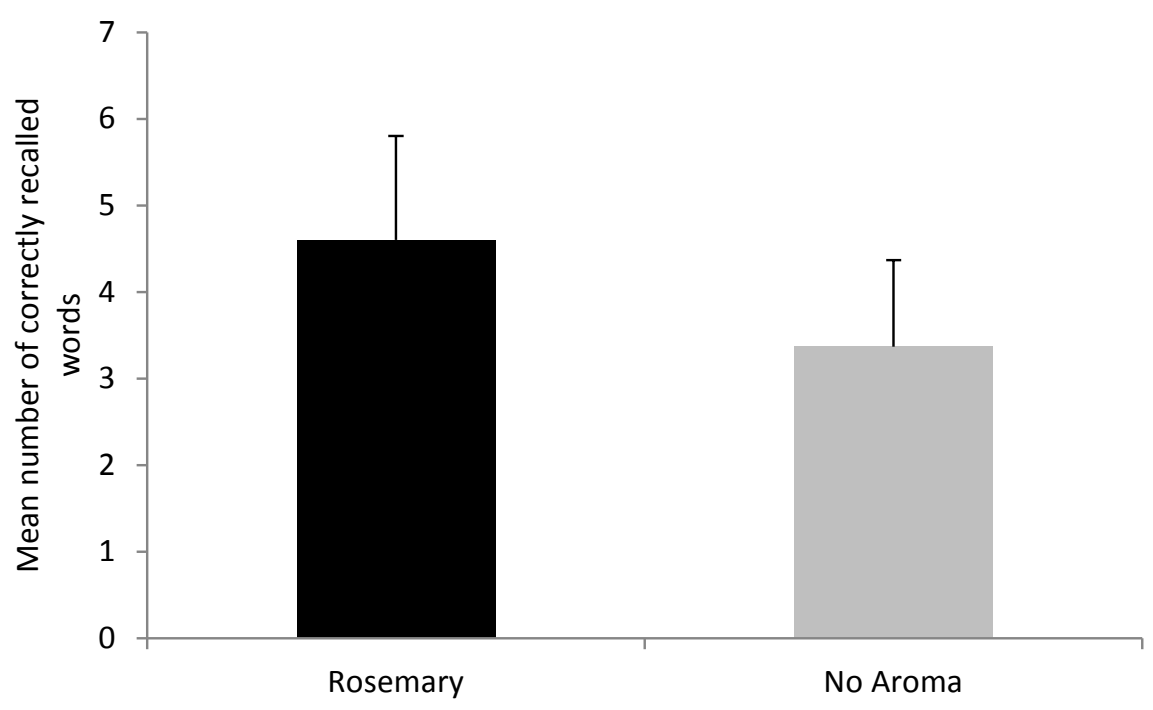

Figure 1. Immediate serial recall. Mean number of correctly recalled words. Error bars represent standard deviations.

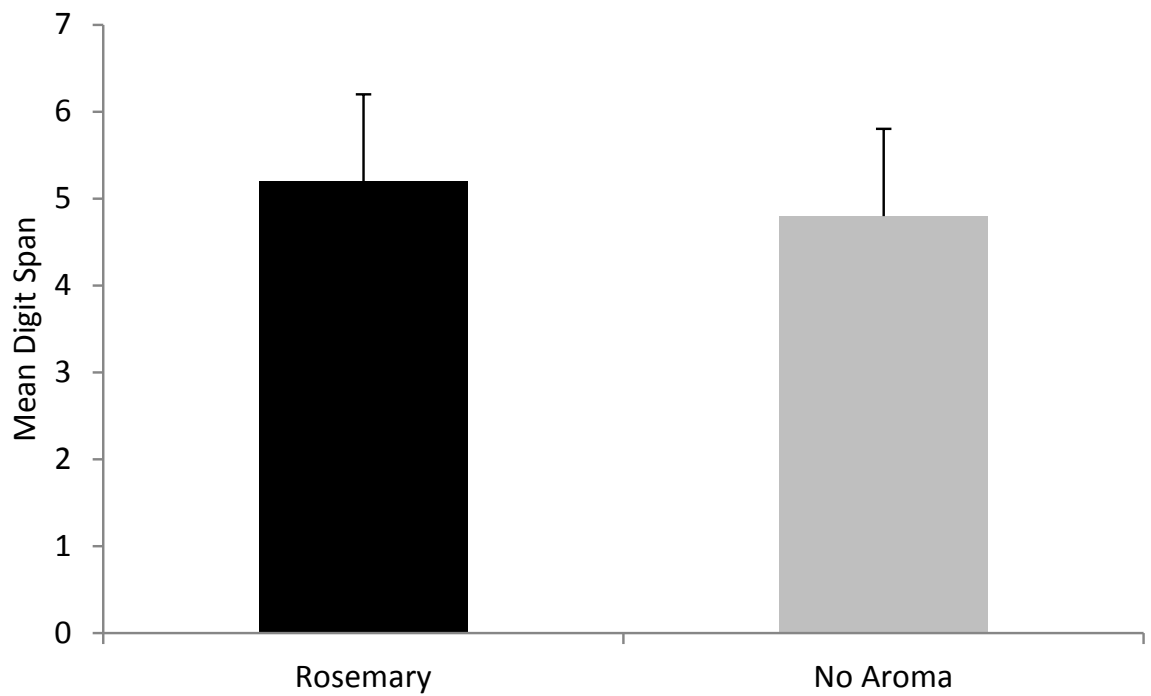

Figure 2. Digit span. Mean correctly recalled digits. Error bars represent standard deviations.

Comparison of the means indicated that children in the Rosemary aroma condition (mean $=4.60$ sentence end words) scored significantly higher than those in the no aroma control condition (mean $=3.37$ sentence end words), Cohen's $\mathrm{d}=$ 0.65 representing a medium to large effect size (Figure 3 ).

\subsection{Counting Span}

Analysis revealed a significant difference between groups $\mathrm{t}(38)=6.91, p<0.05$. Comparison of the means indicated that children in the Rosemary aroma condition (mean $=4.70$ correctly recalled page totals) scored significantly higher than those in the no aroma control condition (mean $=3.80$ correctly recalled page totals), Cohen's $d=0.9$ representing a large effect size (Figure 4 ). 


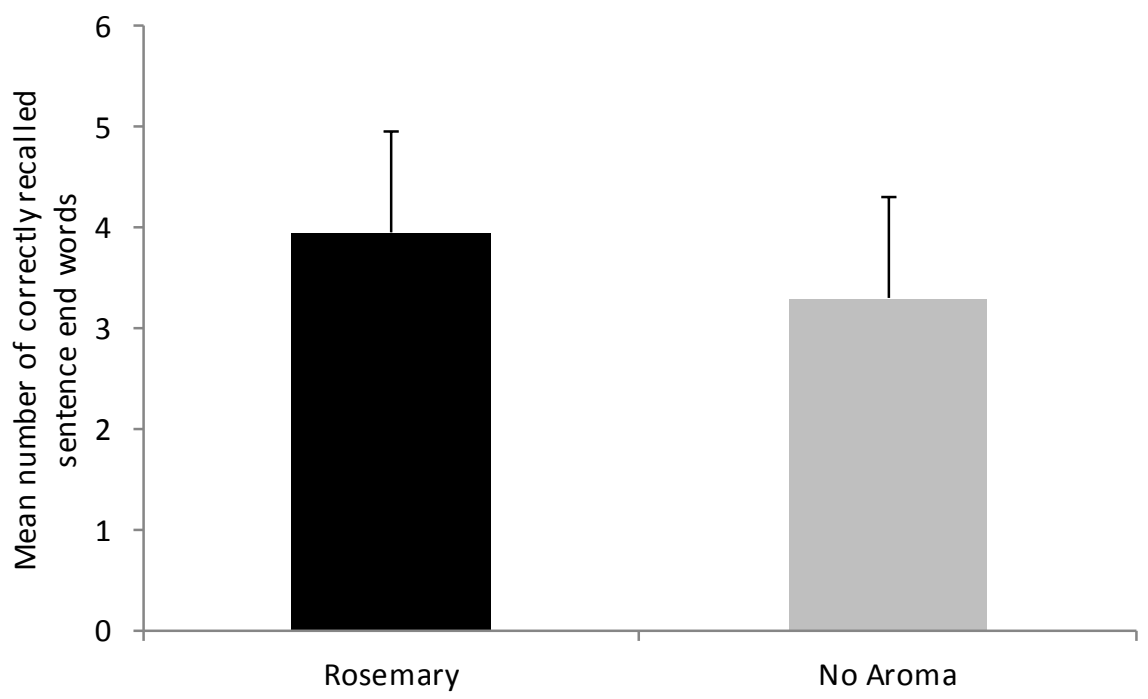

Figure 3. Sentence span. Mean correctly recalled sentence end words. Error bars represent standard deviations.

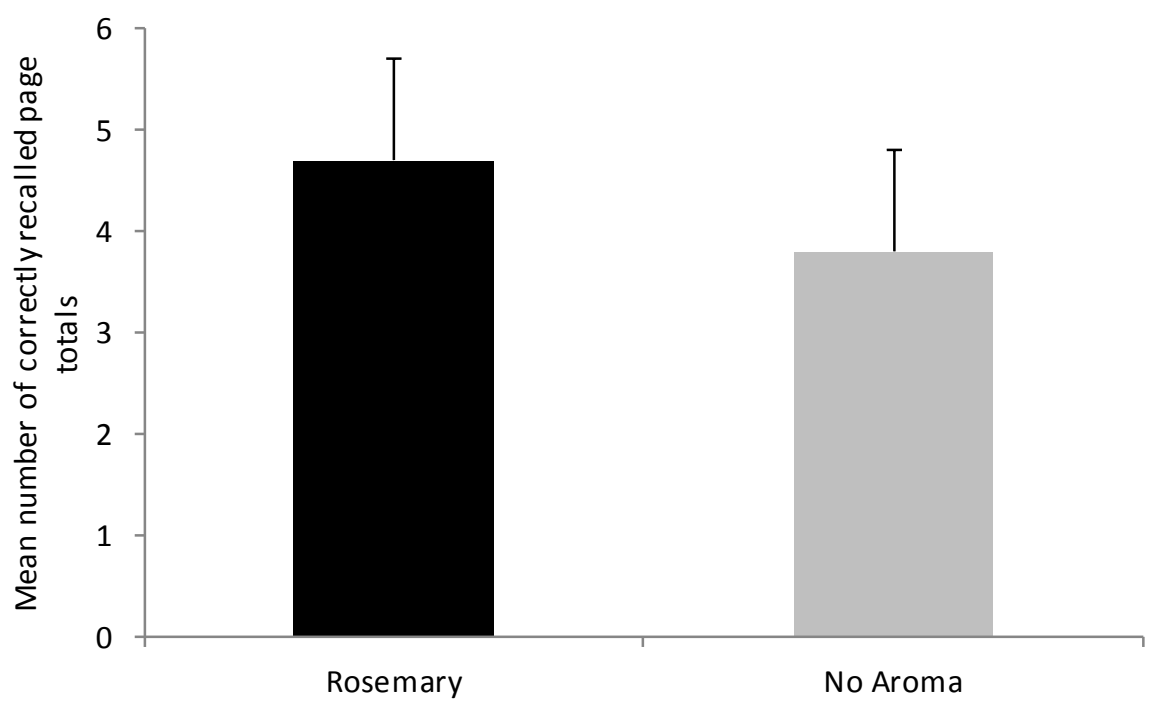

Figure 4. Counting span. Mean correctly recalled page totals. Error bars represent standard deviations.

\subsection{Delayed Word Recall}

Analysis revealed no significant difference between groups $\mathrm{t}(38)=1.45, p=0.15$. Although not significant, comparison of the means indicated that children in the Rosemary aroma condition (mean $=6.35$ words) recalled numerically more correctly than those in the no aroma control condition (mean $=5.60$ words), with a Cohen's $d=0.38$ representing a small to medium effect size (Figure 5 ).

\section{Discussion}

The results of this study show that exposure to the aroma of the essential oil of Rosemary can significantly enhance performance on working memory tasks in children. The effect sizes obtained ranged from small to large based on Cohen's 


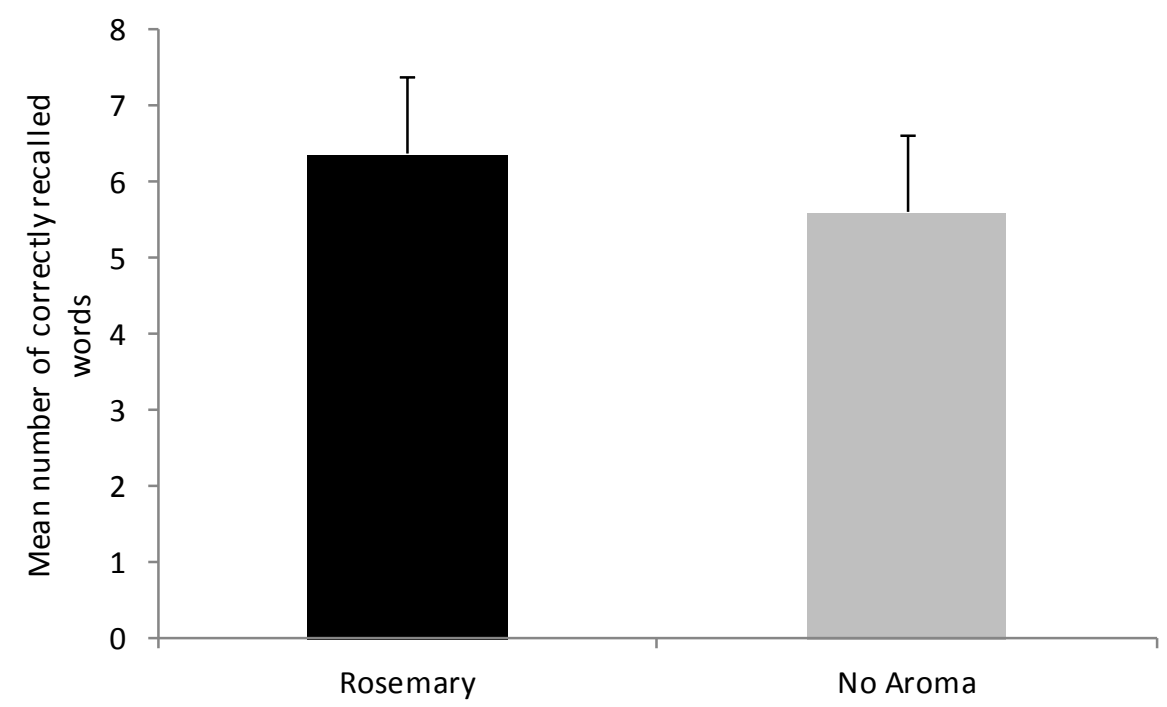

Figure 5. Delayed word recall. Mean correctly recalled words. Error bars represent standard deviations.

criteria [39], with the largest observed for the immediate serial recall and the counting span tasks. Previous studies that have employed healthy adults as the participant sample have reported smaller effect sizes than those observed here [9] [10]. This differential in effects may be the consequence of general age-related changes in physiological and pharmacological sensitivity that have been reported for a range of drugs and toxins [40]. If this is the case it would offer support to the previously proposed pharmacological mechanism that may underpin the activity of Rosemary aroma [41]. However, without further data that permit correlation between serum-based components derived from the essential oil and measures of performance such a conclusion is only tentative, and is not the only plausible explanation of the observed effects. It is possible that a quasi-pharmacological increase in cortical arousal derived from the wide ranging innervations projecting from the olfactory bulb to many areas of the brain [42] improves performance [43].

An important aspect of this work relates to the possible implications of exposure to aromas amongst schoolchildren and their subsequent academic attainment. Previous work [22] [23] [24] has investigated the link between working memory and attainment and suggests the possibility for working memory to be correlated with attainment in Maths English and Science. This raises the intriguing possibility that exposure to the aroma of the essential oil of Rosemary may improve the academic ability of pupils as measured by standardised tests. However, although training in working memory skills has been shown in a number of studies to lead to short term improvements in working memory performance these have not translated into concomitant improvements in tests of attainment [24]. Why the correlation between working memory and attainment does not persist when working memory is improved through training is not clear, although the authors suggest that the nature of the training employed in 
these studies is not transferable to other less directly related tasks. Indeed, they argue that working memory training may produce some modality specific effects on memory processes, whereas working memory includes a domain free executive attention system and it may be this that relates more directly to academic attainment. Of course, the current study did not "train" working memory to achieve improvements. Rather the improvements are seen as a consequence of the exposure to ambient aroma, and a more generalised impact might be evident via an impact on the domain free executive system. This suggestion might support greater transference to attainment. Such a possibility warrants attention and a follow up study should consider measuring academic attainment in tandem with working memory skills including tasks that tap into this system. Interestingly, a personal communication regarding an unpublished study performed in Japan suggests that Maths ability is improved by Rosemary aroma in healthy children [44], although working memory was not included and so the correlation cannot be established.

Beyond the possibility of producing enhancements in cognitive performance and the impact on academic ability lies the question of mechanisms. A number of possibilities have been outlined. The hedonic valence mechanism asserts that the degree of pleasure/displeasure that is gained from an experience defines the moods that emerge from it, and that mood state affects cognitive and behavioural responses. Evidence suggests that hedonic valence is affected by aromas [45], although more recent evidence shows that when this is controlled for aromas including Rosemary still impact on mood [46]. The children in the current were not asked about the perceptual pleasantness of the aroma or their mood so an evaluation of this mechanism cannot be made. We argue however, that this mechanism seems an unlikely candidate for the effects found as they were not equal across all tasks, and the mechanism does not imply any level of domain specificity. The quasi-pharmacological mechanism describes how volatile compounds present in the aromas stimulate the olfactory bulb that then projects innervations throughout the brain [47]. Each aroma would-by virtue of its chemical make up-possess an idiosyncratic pattern of stimulation based on those constituent parts, and the pattern of observed effects may reflect such, with differences in increased activation for each task. However, the current authors favour a third possible mechanism. The pharmacological mechanism suggests that active volatile compounds enter the blood stream via the nasal or lung mucosa. Although the levels of absorbed molecules may be relatively low, such constituent compounds of essential oils have been recorded in the blood of rodents [48], and humans [41] following exposure to vapours of essential oils. Moreover, in the human trial, recorded serum levels of 1,8 cineole (the major volatile constituent of Rosemary essential oil) correlated with cognitive performance for some, but not all tasks included in the study. This correlation is important as in vitro evaluations of 1,8 cineole have shown that it may possess direct pharmacological properties [49], or act as a suitable marker for the absorption of the 
highly active compounds rosmarinic acid [50] and ursolic acid [51] that are found in Rosemary essential oil. In terms of the overall pattern of impact on cognition, we contend that there is the potential for a synergistic effect derived from the presence of all three of these compounds in blood serum as previously reported elsewhere [52]. Given their small size and lipid soluble chemical nature passage across the blood brain barrier would be facilitated [53], permitting direct action at the neuronal receptor site, or indirectly at the enzymatic level. The differential in impact across tasks being a consequence of the involvement of specific neurotransmitter systems that underpin their performance.

Despite active compounds being identified in blood serum following exposure to aromas, the issue of dosing in aroma-based studies is one that consistently proves to be problematic. When aromas are diffused ambiently as in the current study there is little or no control of the amount being delivered to individual participants. Assumptions have to be made regarding equal aroma diffusion in the testing room and similar breathing patterns of participants. Two factors that almost certainly will vary [54] [55]. In order to try to control the dose of aroma being delivered, nasal cannula or face masks attached to a controlled delivery device can be employed. Studies employing such techniques could prove valuable in examining dose response curves and absorption rates. However, the current authors argue that ecological validity of aroma use is an important aspect here, and that the loss of precision in ambiently diffused aroma studies is a bearable cost in order to examine how aromas might influence behaviour when employed in a naturalistic manner.

\section{Conclusion}

In summary, the findings presented here support those of previous work that suggest the aroma of the essential oil of Rosemary can positively influence cognition. The potential for further investigations into controlled atmosphere diffusion is intriguing but possesses a number of ethical implications. For the individual interested in making positive lifestyle interventions, the potential benefits of aromas continue to offer opportunities for improved health, wellbeing and cognition.

\section{References}

[1] Raskin, I., et al. (2002) Plants and Human Health in the Twenty-First Century. Trends in Biotechnology, 20, 522-531. https://doi.org/10.1016/S0167-7799(02)02080-2

[2] Classen, C., Howes, D. and Synnott, A. (1994) Aroma: The Cultural History of Smell. Taylor \& Francis, Oxford.

[3] Rose, J.E. and Behm, F.M. (1994) Inhalation of Vapor from Black Pepper Extract Reduces Smoking Withdrawal Symptoms. Drug and Alcohol Dependence, 34, 225-229. https://doi.org/10.1016/0376-8716(94)90160-0

[4] Foreyt, J.P. and Kennedy, W.A. (1971) Treatment of Overweight by Aversion Therapy. Behaviour Research and Therapy, 9, 29-34. 
[5] Eguchi, E., et al. (2016) The Effects of Aroma Foot Massage on Blood Pressure and Anxiety in Japanese Community-Dwelling Men and Women: A Crossover Randomized Controlled Trial. PloS one, 11, e0151712. https://doi.org/10.1371/journal.pone.0151712

[6] Warm, J.S., Dember, W.N. and Parasuraman, R. (1991) Effects of Olfactory Stimulation on Performance and Stress. Journal of Cosmetic Science, 42, 199-210.

[7] Moss, M., et al. (2008) Modulation of Cognitive Performance and Mood by Aromas of Peppermint and Ylang-Ylang. International Journal of Neuroscience, 118, 59-77. https://doi.org/10.1080/00207450601042094

[8] Lawless, J. (2014) Aromatherapy and the Mind. HarperCollins, UK.

[9] Diego, M.A., et al. (1998) Aromatherapy Positively Affects Mood, Eeg Patterns of Alertness and Math Computations. International Journal of Neuroscience, 96, 217-224. https://doi.org/10.3109/00207459808986469

[10] Moss, M., et al. (2003) Aromas of Rosemary and Lavender Essential Oils Differentially Affect Cognition and Mood in Healthy Adults. International Journal of Neuroscience, 113, 15-38. https://doi.org/10.1080/00207450390161903

[11] Moss, M. (2014) Half Way to Scarborough Fair? The Cognitive and Mood Effects of Rosemary and Sage Aromas. Phytothérapie, 1-6.

[12] Forrester, L.T., et al. (2014) Aromatherapy for Dementia. The Cochrane Library, Hoboken. https://doi.org/10.1002/14651858.CD003150.pub2

[13] Lo, R.S. and Woo, J. (2001) Palliative Care in Old Age. Reviews in Clinical Gerontology, 11, 149-157. https://doi.org/10.1017/S095925980101125X

[14] Sanderson, H. and Ruddle, J. (1992) Aromatherapy and Occupational Therapy. British Journal of Occupational Therapy, 55, 310-314. https://doi.org/10.1177/030802269205500807

[15] Williams, T.I. (2006) Evaluating Effects of Aromatherapy Massage on Sleep in Children with Autism: A Pilot Study. Evidence-Based Complementary and Alternative Medicine, 3, 373-377. https://doi.org/10.1093/ecam/nel017

[16] Ndao, D.H., et al. (2012) Inhalation Aromatherapy in Children and Adolescents Undergoing Stem Cell Infusion: Results of a Placebo-Controlled Double-Blind Trial. Psycho-Oncology, 21, 247-254. https://doi.org/10.1002/pon.1898

[17] Jaafarzadeh, M., Arman, S. and Pour, F.F. (2013) Effect of Aromatherapy with Orange Essential Oil on Salivary Cortisol and Pulse Rate in Children during Dental Treatment: A Randomized Controlled Clinical Trial. Advanced Biomedical Research, 2, 10. https://doi.org/10.4103/2277-9175.107968

[18] Buckle, J. (1999) Use of Aromatherapy as a Complementary Treatment for Chronic Pain. Alternative Therapies in Health and Medicine, 5, 42.

[19] Epple, G. and Herz, R.S. (1999) Ambient Odors Associated to Failure Influence Cognitive Performance in Children. Developmental Psychobiology, 35, 103-107. https://doi.org/10.1002/(SICI)1098-2302(199909)35:2<103::AID-DEV3>3.0.CO;2-4

[20] Czar, K. (2009) The Effects of Aromatherapy on Alertness in an Inclusion Setting. The Corinthian, 10, 7.

[21] Baddeley, A.D. (1990) The Development of the Concept of Working Memory: Implications and Contributions of Neuropsychology. In: Vallar, G. and Shallice, T., Eds., Neuropsychological Impairments of Short-Term Memory, Cambridge University Press, Cambridge, 54-73. https://doi.org/10.1017/CBO9780511665547.004

[22] Gathercole, S.E., et al. (2004) Working Memory Skills and Educational Attainment: 
Evidence from National Curriculum Assessments at 7 and 14 Years of Age. Applied Cognitive Psychology, 18, 1-16. https://doi.org/10.1002/acp.934

[23] St Clair-Thompson, H.L. and Gathercole, S.E. (2006) Executive Functions and Achievements in School: Shifting, Updating, Inhibition, and Working Memory. The Quarterly Journal of Experimental Psychology, 59, 745-759. https://doi.org/10.1080/17470210500162854

[24] Melby-Lervåg, M. and Hulme, C. (2013) Is Working Memory Training Effective? A Meta-Analytic Review. Developmental Psychology, 49, 270. https://doi.org/10.1037/a0028228

[25] Alloway, T.P. (2006) How Does Working Memory Work in the Classroom? Educational Research and Reviews, 1, 134.

[26] Alloway, T.P. (2009) Working Memory, But Not IQ, Predicts Subsequent Learning in Children with Learning Difficulties. European Journal of Psychological Assessment, 25, 92-98. https://doi.org/10.1027/1015-5759.25.2.92

[27] Gathercole, S.E., et al. (2006) Working Memory in Children with Reading Disabilities. Journal of Experimental Child Psychology, 93, 265-281. https://doi.org/10.1016/j.jecp.2005.08.003

[28] Kamijo, K., et al. (2011) The Effects of an Afterschool Physical Activity Program on Working Memory in Preadolescent Children. Developmental Science, 14, 1046-1058. https://doi.org/10.1111/j.1467-7687.2011.01054.x

[29] Richardson, A., et al. (2000) Fatty Acid Deficiency Signs Predict the Severity of Reading and Related Difficulties in Dyslexic Children. Prostaglandins, Leukotrienes and Essential Fatty Acids (PLEFA), 63, 69-74. https://doi.org/10.1054/plef.2000.0194

[30] Rampersaud, G.C., et al. (2005) Breakfast Habits, Nutritional Status, Body Weight, and Academic Performance in Children and Adolescents. Journal of the American Dietetic Association, 105, 743-760. https://doi.org/10.1016/j.jada.2005.02.007

[31] Gajre, N., et al. (2008) Breakfast Eating Habit and Its Influence on Attention-Concentration, Immediate Memory and School Achievement. Indian Pediatrics, $45,824$.

[32] Pivik, R., et al. (2012) Eating Breakfast Enhances the Efficiency of Neural Networks Engaged during Mental Arithmetic in School-Aged Children. Physiology \& Behavior, 106, 548-555. https://doi.org/10.1016/j.physbeh.2012.03.034

[33] Benton, D., Maconie, A. and Williams, C. (2007) The Influence of the Glycaemic Load of Breakfast on the Behaviour of Children in School. Physiology \& Behavior, 92, 717-724. https://doi.org/10.1016/j.physbeh.2007.05.065

[34] Lam, L.F. and Lawlis, T.R. (2016) Feeding the Brain-The Effects of Micronutrient Interventions on Cognitive Performance among School-Aged Children: A Systematic Review of Randomized Controlled Trials. Clinical Nutrition, 36, 1007-1014. https://doi.org/10.1016/j.clnu.2016.06.013

[35] Gathercole, S.E., et al. (2001) Dissociable Lexical and Phonological Influences on Serial Recognition and Serial Recall. The Quarterly Journal of Experimental Psychology: Section A, 54, 1-30. https://doi.org/10.1080/02724980042000002

[36] Baddeley, A.D. (1997) Human Memory: Theory and Practice. Psychology Press, Hove.

[37] Daneman, M. and Carpenter, P.A. (1980) Individual Differences in Working Memory and Reading. Journal of Verbal Learning and Verbal Behavior, 19, 450-466. https://doi.org/10.1016/S0022-5371(80)90312-6 
[38] Gathercole, S.E. and Pickering, S.J. (2000) Assessment of Working Memory in Sixand Seven-Year-Old Children. Journal of Educational Psychology, 92, 377. https://doi.org/10.1037/0022-0663.92.2.377

[39] Cohen, J. (1992) A Power Primer. Psychological Bulletin, 112, 155. https://doi.org/10.1037/0033-2909.112.1.155

[40] Scheuplein, R., Charnley, G. and Dourson, M. (2002) Differential Sensitivity of Children and Adults to Chemical Toxicity: I. Biological Basis. Regulatory Toxicology and Pharmacology, 35, 429-447. https://doi.org/10.1006/rtph.2002.1558

[41] Moss, M. and Oliver, L. (2012) Plasma 1,8-Cineole Correlates with Cognitive Performance Following Exposure to Rosemary Essential Oil Aroma. Therapeutic Advances in Psychopharmacology, 2, 103-113. https://doi.org/10.1177/2045125312436573

[42] Shipley, M.T. and Ennis, M. (1996) Functional Organization of Olfactory System. Developmental Neurobiology, 30, 123-176. https://doi.org/10.1002/(SICI)1097-4695(199605)30:1<123::AID-NEU11>3.0.CO;2N

[43] Robbins, T. (2000) From Arousal to Cognition: The Integrative Position of the Prefrontal Cortex. Progress in Brain Research, 126, 469-483. https://doi.org/10.1016/S0079-6123(00)26030-5

[44] Fuji, M. (2017) Rosemary Aroma Improves Mathematics Performance in Healthy Children and Adults. Japan, 1.

[45] Baron, R.A. and Thomley, J. (1994) A Whiff of Reality. Environment and Behavior, 26, 766-784. https://doi.org/10.1177/0013916594266003

[46] Burnett, K.M., Solterbeck, L.A. and Strapp, C.M. (2004) Scent and Mood State Following an Anxiety-Provoking Task. Psychological Reports, 95, 707-722. https://doi.org/10.2466/pr0.95.2.707-722

[47] Jellinek, J.S. (1998) Odours and Mental States. International Journal of Aromatherapy, 9, 115-120. https://doi.org/10.1016/S0962-4562(98)80005-2

[48] Jirovetz, L., et al. (1992) Analysis of Fragrance Compounds in Blood Samples of Mice by Gas Chromatography, Mass Spectrometry, GC/FTIR and GC/AES after Inhalation of Sandalwood Oil. Biomedical Chromatography, 6, 133-134. https://doi.org/10.1002/bmc.1130060307

[49] Perry, N.S., et al. (2000) In-Vitro Inhibition of Human Erythrocyte Acetylcholinesterase by Salvia lavandulaefolia Essential Oil and Constituent Terpenes. Journal of Pharmacy and Pharmacology, 52, 895-902. https://doi.org/10.1211/0022357001774598

[50] Orhan, I., et al. (2008) Inhibitory Effect of Turkish Rosmarinus officinalis L. on Acetylcholinesterase and Butyrylcholinesterase Enzymes. Food Chemistry, 108, 663-668. https://doi.org/10.1016/j.foodchem.2007.11.023

[51] Chung, Y.-K., et al. (2001) Inhibitory Effect of Ursolic Acid Purified from Origanum majorana L. on the Acetylcholinesterase. Molecules \& Cells (Springer Science \& Business Media BV), 11, 137-143.

[52] Savelev, S., et al. (2003) Synergistic and Antagonistic Interactions of Anticholinesterase Terpenoids in Salvia lavandulaefolia Essential Oil. Pharmacology Biochemistry and Behavior, 75, 661-668. https://doi.org/10.1016/S0091-3057(03)00125-4

[53] Boyle, R.R., et al. (2005) Rapid Absorption of Dietary 1,8-Cineole Results in Critical Blood Concentration of Cineole and Immediate Cessation of Eating in the Common Brushtail Possum (Trichosurus vulpecula). Journal of Chemical Ecology, 31, 
2775-2790. https://doi.org/10.1007/s10886-005-8393-0

[54] Benchetrit, G. (2000) Breathing Pattern in Humans: Diversity and Individuality. Respiration Physiology, 122, 123-129.

https://doi.org/10.1016/S0034-5687(00)00154-7

[55] Paterson, S. and Mackay, D. (1985) The Fugacity Concept in Environmental Modelling, in Reactions and Processes. Springer, Berlin, 121-140.

https://doi.org/10.1007/978-3-540-39048-0_4 